\title{
CONVOLUTION ESTIMATES AND MODEL SURFACES OF LOW CODIMENSION
}

\author{
DANIEL M. OBERLIN
}

\begin{abstract}
For $k \geq d / 2$ we give examples of measures on $k$-surfaces in $\mathbb{R}^{d}$. These measures satisfy convolution estimates which are nearly optimal.
\end{abstract}

Suppose that $S$ is a smooth $k$-dimensional surface in $\mathbb{R}^{d}$ and that $\mu$ is a smooth positive Borel measure on $S$. Suppose further that $\mu$ satisfies the convolution estimate

$$
\|\mu * f\|_{L^{q}\left(\mathbb{R}^{d}\right)} \leq C\|f\|_{L^{p}\left(\mathbb{R}^{d}\right)}
$$

where the norms are computed using Lebesgue measure $m_{d}$ on $\mathbb{R}^{d}$. Then it is well-known that $p \leq q$. Convolution with the characteristic function of a small ball shows that $(1 / p, 1 / q)$ must lie in the triangle $\Delta(k, d)$ with vertices $(0,0),(1,1)$, and $(d /(2 d-k),(d-k) /(2 d-k))$. And a result of Ricci ([5]), which extends an observation of Carbery and Christ, shows that if $k(k+3)<2 d$, then (10) also implies that

$$
\frac{1}{p}-\frac{1}{q} \leq \frac{2 k}{6 d-k^{2}-5 k} \text {. }
$$

Let $\mathcal{T}(k, d)$ be $\Delta(k, d)$ if $k(k+3) \geq 2 d$ and the subset of $\Delta(k, d)$ defined by (2) if $k(k+3)<2 d$. Suppose now that $S$ has the form

$$
\left\{\left(y ; \Phi_{1}(y), \Phi_{2}(y), \ldots, \Phi_{l}(y)\right): y \in G\right\}
$$

where $G$ is a nonempty open subset of $\mathbb{R}^{k}$, where $l=d-k$, and where the functions $\Phi_{j}: \mathbb{R}^{k} \rightarrow \mathbb{R}$ are homogeneous polynomials. Let $\mu$ be the measure on $S$ induced by $m_{k}$ on $G$. Then we will say that $S$ is a model surface if (11) holds whenever $(1 / p, 1 / q)$ lies in the interior of $\mathcal{T}(k, d)$.

\section{Examples:}

(i) the paraboloids $\left\{\left(y ;|y|^{2}\right): y \in \mathbb{R}^{d-1},|y|<1\right\}$ (see, e.g., pp. 370-371 in [6]);

(ii) the moment curves $\left\{\left(y ; y^{2}, \ldots, y^{d}\right): 0<y<1\right\}$ (see [1]);

(iii) the monomial surfaces $\left\{\left(y ; \Phi_{1}(y), \ldots, \Phi_{l}(y)\right): y \in \mathbb{R}^{k},|y|<1\right\}$ where $l=k+\frac{k(k-1)}{2}$ and the functions $\Phi_{j}$ are the distinct quadratic monomials (see [5]);

Date: June, 2007.

1991 Mathematics Subject Classification. 42B10.

Key words and phrases. measure, convolution estimate.

The author was supported in part by NSF grant DMS-0552041. 

[4]);

(iv) the 3 -surface $\left\{\left(y_{1}, y_{2}, y_{3} ; y_{1}^{2}+y_{2}^{2}, y_{2}^{2}+y_{3}^{2}\right): 0<y_{j}<1\right\}$ in $\mathbb{R}^{5}$ (see

(v) certain surfaces of the form $\left\{\left(y ; \Phi_{1}(y), \ldots, \Phi_{l}(y)\right): y \in \mathbb{R}^{k},|y|<1\right\}$ where $l=k$ (see [2]).

Of course most polynomial surfaces $S$ of the form (3) are not model surfaces in our sense: the convolution requirement rules out degeneracies which result from the presence of "flatness" or the lack of "curvature". When $k=1$ or $k=d-1$ there are obvious and simple technical interpretations of "curvature". In a few other cases there are technical interpretations which are neither obvious nor simple. For example, when $k=2$ and $d=4$ the interpretation is that

$$
\begin{aligned}
\left(\Phi_{1 y_{1} y_{1}} \Phi_{2 y_{1} y_{2}}-\Phi_{2 y_{1} y_{1}} \Phi_{1 y_{1} y_{2}}\right) & \left(\Phi_{2 y_{2} y_{2}} \Phi_{1 y_{1} y_{2}}-\Phi_{2 y_{1} y_{2}} \Phi_{1 y_{2} y_{2}}\right) \\
& -\left(\left(\Phi_{1 y_{1} y_{1}} \Phi_{2 y_{2} y_{2}}-\Phi_{2 y_{1} y_{1}} \Phi_{1 y_{2} y_{2}}\right)\right)^{2}
\end{aligned}
$$

not vanish. At any rate, the examples mentioned above, along with certain of their Cartesian products, constitute a fairly complete list of the known model surfaces. The aim of this note is to extend that list by providing examples of model surfaces whenever $k \geq \frac{d}{2}$.

Fix positive integers $k$ and $l$ with $1 \leq l \leq k$ and put $d=k+l$. Let $\mathcal{C}=\left[c_{i}^{j}\right]$ be a $k$ by $l$ matrix of real numbers. For $1 \leq j \leq l$ define bilinear forms $L_{j}: \mathbb{R}^{k} \times \mathbb{R}^{k} \rightarrow \mathbb{R}$ by

$$
L_{j}(x, y)=\sum_{i=1}^{k} c_{i}^{j} x_{i} y_{i}
$$

and put $\Phi_{j}(y)=L_{j}(y, y)$. We will say that $\mathcal{C}$ satisfies condition $\left(^{*}\right)$ if every $l$ by $l$ submatrix of $\mathcal{C}$ is nonsingular.

Theorem. With the $\Phi_{j}$ as above, with $G=B(0,1)$, and with $S$ given by (3), suppose that $\left(^{*}\right)$ holds. Then $S$ is a model surface.

Proof of theorem: Since $k(k+3) \geq 2 d$, it is required to establish (11) whenever $\left(\frac{1}{p}, \frac{1}{q}\right)$ lies in the interior of $\Delta(k, d)$. With $q_{0}=\frac{2 d-k}{d-k}$, an interpolation argument shows that it is suffices to prove that

$$
\left\|\mu * \chi_{E}\right\|_{q_{0}} \leq C(p) m_{d}(E)^{1 / p}
$$

for measurable $E \subset \mathbb{R}^{d}$ and $p>\frac{2 d-k}{d}$. And, since $\mu$ has compact support, we can also assume that $E \subset B(0,1)$. For such $E$, (44) will follow, as in [4], from the auxiliary inequality (6) below. Thus, writing $\Phi(y)=$ $\left(y ; \Phi_{1}(y), \ldots, \Phi_{l}(y)\right)$,

$(5)$

$$
\begin{aligned}
& \left\|\mu * \chi_{E}\right\|_{q_{0}}^{q_{0}} \\
& =\int_{\mathbb{R}^{d}} \int_{B(0,1)} \chi_{E}(z-\Phi(x)) d m_{k}(x)\left(\int_{B(0,1)} \chi_{E}(z-\Phi(y)) d m_{k}(y)\right)^{q_{0}-1} d m_{d}(z) \\
& =\int_{\mathbb{R}^{d}} \chi_{E}(z) \int_{B(0,1)}\left(\int_{B(0,1)} \chi_{E}(z+\Phi(x)-\Phi(y)) d m_{k}(y)\right)^{q_{0}-1} d m_{k}(x) d m_{d}(z) .
\end{aligned}
$$


Now assume, for the moment, the inequality

$$
\left(\int_{B(0,1)}\left[\int_{B(0,1)} \chi_{\widetilde{E}}(\Phi(x)-\Phi(y)) d m_{k}(y)\right]^{d / l} d m_{k}(x)\right)^{l / d} \leq C(\widetilde{p}) m_{d}(\widetilde{E})^{1 / \widetilde{p}}
$$

for $\widetilde{p}>\frac{d}{k}$ and $\widetilde{E} \subset B(0,2)$. Since $q_{0}-1=\frac{d}{l}$, (5) and (6) yield

$$
\left\|\mu * \chi_{E}\right\|_{q_{0}} \leq C(\widetilde{p}) m_{d}(E)^{\left(1+\frac{1}{\tilde{p}} \frac{d}{l}\right) \frac{1}{q_{0}}} .
$$

If $\frac{1}{p}=\left(1+\frac{1}{\tilde{p}} \frac{d}{l}\right) \frac{1}{q_{0}}$, then $\widetilde{p}>\frac{d}{k}$ if and only if $p>\frac{2 d-k}{d}$. Thus, as claimed, (44) will follow from ([6). Now (6) is equivalent to the inequality, for nonnegative $f$,

$$
\begin{array}{r}
\int_{B(0,1)} \int_{B(0,1)} f(x) \chi_{\widetilde{E}}\left(x-y ; \sum c_{i}^{1}\left(x_{i}^{2}-y_{i}^{2}\right), \ldots, \sum c_{i}^{l}\left(x_{i}^{2}-y_{i}^{2}\right)\right) d m_{k}(y) d m_{k}(x) \\
\leq C(\widetilde{p})\|f\|_{L^{d / k}\left(\mathbb{R}^{k}\right)} m_{d}(\widetilde{E})^{1 / \widetilde{p}}
\end{array}
$$

where $\sum$ means $\sum_{i=1}^{k}$. In the $y$-integral we change variables to obtain

$$
\int_{B(0,1)} \int_{B(0,1)} f(x) \chi_{\widetilde{E}}\left(y ; \sum c_{i}^{1}\left(2 x_{i} y_{i}-y_{i}^{2}\right), \ldots, \sum c_{i}^{l}\left(2 x_{i} y_{i}-y_{i}^{2}\right)\right) d m_{k}(y) d m_{k}(x) .
$$

If $E \subset \mathbb{R}^{d}$ is defined by

$$
\chi_{E}\left(y_{1}, \ldots, y_{k} ; u_{1}, \ldots, u_{l}\right)=\chi_{\widetilde{E}}\left(y_{1}, \ldots, y_{k}, 2 u_{1}-\sum c_{i}^{1} y_{i}^{2}, \ldots, 2 u_{l}-\sum c_{i}^{l} y_{i}^{2}\right),
$$

then $m_{d}(E)=2^{-l} m_{d}(\widetilde{E})$ and the left hand side of (7) may be written

$$
\int_{B(0,1)} \int_{B(0,1)} f(x) \chi_{E}\left(y ; L_{1}(x, y), \ldots, L_{l}(x, y)\right) d m_{k}(y) d m_{k}(x)
$$

Thus (7) will follow from

$$
\int_{B(0,1)} \int_{B(0,1)} f(x) \chi_{E}\left(y ; L_{1}(x, y), \ldots, L_{l}(x, y)\right) d m_{k}(y) d m_{k}(x) \leq C\|f\|_{L^{d / k}\left(\mathbb{R}^{k}\right)} m_{d}(E)^{1 / \widetilde{p}}
$$

whenever $f$ is nonnegative, $\widetilde{p}>\frac{d}{k}$, and $E \subset B(0,2)$. (The constant $C$ will depend on $\widetilde{p}$ and $\mathcal{C}$.) For an multi-index $\mathbf{n}=\left(n_{1}, \ldots, n_{k}\right)$ we will write $\left\{\left|y_{i}\right| \sim 2^{n_{i}}\right\}$ to stand for the set of $y \in \mathbb{R}^{k}$ for which the $k$ inequalities $2^{n_{i}} \leq\left|y_{i}\right|<2^{n_{i}+1}$ hold. Our main task will be to establish the estimate (9)

$$
\begin{aligned}
\int_{\mathbb{R}^{k}} \int_{\left\{\left|y_{i}\right| \sim 1\right\}} f(x) \chi_{E}\left(y ; L_{1}(x, y), \ldots, L_{l}(x, y)\right) & d m_{k}(y) d m_{k}(x) \\
& \leq C\|f\|_{L^{d / k}\left(\mathbb{R}^{k}\right)} m_{d}(E)^{k / d}
\end{aligned}
$$


for all nonnegative $f$ and $E \subset \mathbb{R}^{d}$. From this a change of variables shows that the inequalities

(10)

$$
\begin{aligned}
\int_{\mathbb{R}^{k}} \int_{\left\{\left|y_{i}\right| \sim 2^{\left.n_{i}\right\}}\right.} f(x) \chi_{E}\left(y ; L_{1}(x, y), \ldots, L_{l}(x, y)\right) & d m_{k}(y) d m_{k}(x) \\
& \leq C\|f\|_{L^{d / k}\left(\mathbb{R}^{k}\right)} m_{d}(E)^{k / d}
\end{aligned}
$$

hold uniformly in $\mathbf{n}$. This implies (8): suppose $E \subset[-2,2]^{d}$. For a multiindex $\mathbf{n}$ let $E_{\mathbf{n}}$ be the set of $\left(y_{1}, \ldots, y_{k} ; u_{1}, \ldots, u_{l}\right) \in E$ for which $\left|y_{i}\right| \sim 2^{n_{i}}$. Then $m_{d}\left(E_{\mathbf{n}}\right) \leq 2^{\sum_{i}\left(n_{i}+1\right)}$ and so, if $\frac{1}{\widetilde{p}}=\frac{k}{d}-\epsilon$,

$$
m_{d}\left(E_{\mathbf{n}}\right)^{k / d} \leq m_{d}(E)^{1 / \widetilde{p}} 2^{\epsilon \sum_{i}\left(n_{i}+1\right)} .
$$

Applying (10) with $E$ replaced by $E_{\mathbf{n}}$ and then summing over $\mathbf{n}$ for which $-\infty<n_{j} \leq 0$ yields (요).

Moving to the proof of (9), we write, for suitable functions $g$ on $\mathbb{R}^{d}$, (11)

$$
\begin{array}{r}
\int_{\mathbb{R}^{k}} \int_{\left\{\left|y_{i}\right| \sim 1\right\}} f(x) g\left(y ; L_{1}(x, y), \ldots, L_{l}(x, y)\right) d m_{k}(y) d m_{k}(x)=\langle T f, g\rangle \\
=\int_{\mathbb{R}^{l}} \int_{\left\{\left|y_{i}\right| \sim 1\right\}} T f(y ; u) g(y ; u) d m_{k}(y) d m_{l}(u) .
\end{array}
$$

Then (9) is a consequence of the fact, which we will establish below, that

$$
T: L^{d / k}\left(\mathbb{R}^{k}\right) \rightarrow L^{d / l}\left(\mathbb{R}^{d}\right)
$$

Although it does not figure here, one can regard the operator $T$ as a restricted $(k-l)$-plane transform operating on a function $f$ defined on $\mathbb{R}^{k}$ by integrating $f$ over the $(k-l)$-plane

$$
\left\{x \in \mathbb{R}^{k}: L_{1}(x, y)=u_{1}, \ldots, L_{l}(x, y)=u_{l}\right\} .
$$

Since the indices in (12) are conjugate, it is natural to attempt to prove (12) by embedding $T$ in an analytic family of operators $\left\{T_{z}\right\}$ and then interpolating between $L^{1} \rightarrow L^{\infty}$ and $L^{2} \rightarrow L^{2}$ estimates. Thus we define

$$
T_{z} f(y ; u)=C(z) f(y ; \cdot) *|\cdot|^{z}(u),
$$

where the convolution is in the $u$ variable and $C(z)$ is chosen to compensate for the singularities of the distributions $|\cdot|^{z}$ on $\mathbb{R}^{l}-$ see p. 363 in [3]. Next we will observe that

$$
\left\|T_{z} f\right\|_{L^{\infty}\left(\mathbb{R}^{d}\right)} \leq c_{0}(y)\|f\|_{L^{1}\left(\mathbb{R}^{k}\right)}
$$

if $z=0+i s$ and then prove (using the hypothesis $(*)$ ) that

$$
\left\|T_{z} f\right\|_{L^{2}\left(\mathbb{R}^{d}\right)} \leq c_{1}(y)\|f\|_{L^{2}\left(\mathbb{R}^{k}\right)}
$$

if $z=-\frac{d}{2}+i s$. 
Note that (11) implies that $T f(y ; u)=0$ unless $\left|y_{j}\right| \sim 1$. If $\left|y_{j}\right| \sim 1$ we will need the following formula:

$$
\int_{\mathbb{R}^{l}} T f(y ; u) h(u) d m_{l}(u)=\int_{\mathbb{R}^{k}} f(x) h\left(L_{1}(x, y), \ldots, L_{l}(x, y)\right) d m_{k}(x),
$$

valid for nice functions $h$ on $\mathbb{R}^{l}$. To see (15) with $y=\widetilde{y}$, fix $\widetilde{y}$ with $\left|\widetilde{y}_{i}\right| \sim 1$, take $g(y ; u)=\chi_{B(\widetilde{y}, \delta)}(y) h(u)$ in the extreme terms of (11), and then let $\delta \rightarrow 0$.

Now (13) follows immediately from (15).

To prove (14) we start by setting some notation. For fixed $y \in \mathbb{R}^{k}$ we consider the mapping $\mathcal{L}_{y}$ of $\mathbb{R}^{k}$ into $\mathbb{R}^{l}$ defined by

$$
\mathcal{L}_{y} x=\left(L_{1}(x, y), \ldots, L_{l}(x, y)\right)
$$

along with the adjoint map $\mathcal{L}_{y}^{*}$ of $\mathbb{R}^{l}$ to $\mathbb{R}^{k}$ defined by

$$
\left\langle\mathcal{L}_{y}^{*} \zeta, x\right\rangle=\left\langle\zeta, \mathcal{L}_{y} x\right\rangle
$$

Then (15) implies that

$$
\widehat{T f(y, \cdot)}(\zeta)=\widehat{f}\left(\mathcal{L}_{y}^{*} \zeta\right)
$$

In order to prove (14) by exploiting (16), we need a lemma.

Lemma. Under the assumption $\left({ }^{*}\right)$ on $\mathcal{C}$, there is $c$, depending on $\mathcal{C}$ and $\rho \in \mathbb{R}$, such that the inequality

$$
\int_{\left\{\left|y_{j}\right| \sim 1\right\}} \int_{\mathbb{R}^{l}}|\zeta|^{\rho} w\left(\mathcal{L}_{y}^{*} \zeta\right) d m_{l}(\zeta) d m_{k}(y) \leq c \int_{\mathbb{R}^{k}}|\tau|^{\rho-k+l} w(\tau) d m_{k}(\tau)
$$

holds for nonnegative functions $w$ on $\mathbb{R}^{k}$.

Proof of Lemma: If $x, y \in \mathbb{R}^{k}$, we may write $x(i)$ instead of $x_{i}$ and $x y$ to stand for the vector with $x y(i)=x(i) y(i)$. Let 1 stand for the vector $(1,1, \ldots, 1)$. One may check that, for $i=1, \ldots, k, \mathcal{L}_{1}^{*} \zeta(i)=\sum_{j} c_{i}^{j} \zeta_{j}$ and also that $\mathcal{L}_{y}^{*} \zeta=y \mathcal{L}_{1}^{*} \zeta$. In particular, the hypothesis $\left({ }^{*}\right)$ on $\mathcal{C}$ has the following interpretation in terms of the $\left(\begin{array}{l}k \\ l\end{array}\right)$ coordinate projections $\pi$ of $\mathbb{R}^{k}$ onto $\mathbb{R}^{l}$ : for each such $\pi, \pi \circ \mathcal{L}_{1}^{*}: \mathbb{R}^{l} \rightarrow \mathbb{R}^{l}$ is nonsingular. It follows that there is $M<\infty$ such that if $P \subset\{1, \ldots, k\}$ satisfies $|P|=l$, then

$$
|\zeta| \leq M \sup _{i \in P}\left|\mathcal{L}_{1}^{*} \zeta(i)\right| \text {. }
$$

Next note that if $\zeta \in \mathbb{R}^{l}$, then there are $1 \leq i_{l+1}<i_{l+2}<\cdots<i_{k} \leq k$, dependent on $\zeta$, such that

$$
|\zeta| \leq M\left|\mathcal{L}_{1}^{*} \zeta\left(i_{a}\right)\right| \text { if } a=l+1, \ldots, k .
$$

(Having $|\zeta|>M\left|\mathcal{L}_{1}^{*} \zeta(i)\right|$ for even $l i$ 's would contradict (18).) In this situation, write $Q=\left\{i_{l+1}, \ldots, i_{k}\right\}$ and $\zeta \in F_{Q}$ so that $\mathbb{R}^{l}=\cup_{Q} F_{Q}$, where the union is taken over all $Q \subset\{1, \ldots, k\}$ such that $|Q|=k-l$. Then (17) will follow by summing over $Q$ the estimates

$$
\int_{\left\{\left|y_{i}\right| \sim 1\right\}} \int_{F_{Q}}|\zeta|^{\rho} w\left(\mathcal{L}_{y}^{*} \zeta\right) d m_{l}(\zeta) d m_{k}(y) \leq c \int_{\mathbb{R}^{k}}|\tau|^{\rho-k+l} w(\tau) d m_{k}(\tau)
$$


To establish (20), fix first $Q=\left\{i_{l+1}, \ldots, i_{k}\right\}$, then $i_{1}, \ldots, i_{l}$ with $\left\{i_{1}, \ldots, i_{k}\right\}=$ $\{1, \ldots, k\}$, and finally $y_{i_{1}}, \ldots, y_{i_{l}}$ with $\left|y_{i_{a}}\right| \sim 1$. Consider the map

$$
\left(\zeta_{1}, \ldots, \zeta_{l}, y_{i_{l+1}}, \ldots, y_{i_{k}}\right) \mapsto \tau \doteq\left(y_{i_{1}} \mathcal{L}_{1}^{*} \zeta\left(i_{1}\right), \ldots, y_{i_{k}} \mathcal{L}_{1}^{*} \zeta\left(i_{k}\right)\right) \simeq \mathcal{L}_{y}^{*} \zeta
$$

where the $\simeq$ indicates a permutation of the coordinates. We want to estimate the absolute value $J$ of the Jacobian determinant of (21) when $\zeta \in F_{Q}$. To do this, write $\tau$ as

$$
\left(y_{i_{1}} \sum c_{i_{1}}^{j} \zeta_{j}, \ldots, y_{i_{l}} \sum c_{i_{l}}^{j} \zeta_{j}, y_{i_{l+1}} \sum c_{i_{l+1}}^{j} \zeta_{j}, \ldots, y_{i_{k}} \sum c_{i_{k}}^{j} \zeta_{j}\right)
$$

where $\sum$ means $\sum_{j=1}^{l}$. Computing the Jacobian matrix, one sees that

$$
J=\prod_{a=1}^{l}\left|y_{i_{a}}\right| \times\left|D\left(i_{1}, \ldots, i_{l}\right)\right| \times \prod_{a=l+1}^{k}\left|\mathcal{L}_{1}^{*} \zeta\left(i_{a}\right)\right|,
$$

where $D\left(i_{1}, \ldots, i_{l}\right)$ is the determinant of the $l$ by $l$ matrix obtained by retaining only the rows of $\mathcal{C}$ corresponding to $i=i_{1}, \ldots, i_{l}$. By $\left(^{*}\right),\left|D\left(i_{1}, \ldots, i_{l}\right)\right| \geq$ $c(\mathcal{C})>0$. Since $\zeta \in F_{Q},\left|\mathcal{L}_{1}^{*} \zeta\left(i_{a}\right)\right| \geq \frac{|\zeta|}{M}$ for $a=l+1, \ldots, k$. It then follows from $\left|y_{i}\right| \sim 1$ that

$$
J \geq c|\zeta|^{k-l}
$$

It is also easy to check (see (18) ) that $|\zeta|^{\rho} \leq c\left|\mathcal{L}_{1}^{*} \zeta\right|^{\rho}$. So the inequality

$$
\int_{\left\{\left|y_{i}\right| \sim 1\right\}} \int_{F_{Q}}|\zeta|^{\rho} w\left(\mathcal{L}_{y}^{*} \zeta\right) d m_{l}(\zeta) d y_{i_{l+1}} \cdots d y_{i_{k}} \leq c \int_{\mathbb{R}^{k}}|\tau|^{\rho-k+l} w(\tau) d m_{k}(\tau)
$$

follows by change of variables, and then (20) follows by integrating with respect to $y_{i_{1}}, \ldots, y_{i_{l}}$ (since $\left|y_{i}\right| \sim 1$ ). This concludes the proof of the lemma.

With (17) we can prove (14): suppose $z=-\frac{d}{2}+i s$. Then

$$
\begin{aligned}
\int_{\left\{\left|y_{i}\right| \sim 1\right\}} \int_{\mathbb{R}^{l}}\left|T_{z} f(y, u)\right|^{2} & \left.d m_{l}(u) d m_{k}(y)=\int_{\left\{\left|y_{i}\right| \sim 1\right\}} \int_{\mathbb{R}^{l}} \mid \widehat{T_{z} f(y, \cdot)}\right)\left.(\zeta)\right|^{2} d m_{l}(\zeta) d m_{k}(y) \\
& =\left.\left.c(s) \int_{\left\{\left|y_{i}\right| \sim 1\right\}} \int_{\mathbb{R}^{l}}\left|\widehat{f}\left(\mathcal{L}_{y}^{*} \zeta\right)\right|^{2}|| \zeta\right|^{d / 2-l}\right|^{2} d m_{l}(\zeta) d m_{k}(y) \\
& \leq c(s) \int_{\mathbb{R}^{k}}|\widehat{f}(\tau)|^{2}|\tau|^{d-2 l-(k-l)} d m_{k}(\tau)=c(s)\|f\|_{L^{2}\left(\mathbb{R}^{k}\right)}^{2}
\end{aligned}
$$

where: the second equality follows from (16) and the fact that, on $\mathbb{R}^{l}$, $\widehat{|\cdot|^{z}}(\zeta)=c(z)|\zeta|^{-z-l}([3]$, p. 363); the inequality follows from (17); and the last equality follows from $d=k+l$. This proves (14). Now interpolating between (13) and (14) shows that

$$
T_{z}: L^{d / k}\left(\mathbb{R}^{k}\right) \rightarrow L^{d / l}\left(\mathbb{R}^{d}\right)
$$

if $z=-l+i s$. Since $T_{-l}$ is a scalar multiple of $T$, (12) follows, concluding the proof of the theorem. 


\section{REFERENCES}

[1] M. Christ, Convolution, curvature, and combinatorics: a case study, Internat. Math. Res. Notices 19 (1998), 1033-1048.

[2] S.W. Drury and K. Guo, Convolution estimates related to surfaces of half the ambient dimension, Math. Proc. Cambridge Phil. Soc. 110 (1991), 151-159.

[3] I.M. Gelfand and G.E. Shilov, Generalized Functions, vol. I, Academic Press, New York, 1964.

[4] D. Oberlin, Convolution and restriction for a 3 -surface in $\mathbb{R}^{5}$, J. of Fourier Analysis and Applications 10 (2004), pp. 377-382.

[5] F. Ricci, $L^{p}-L^{q}$ boundedness for convolution operators defined by singular measures in $\mathbb{R}^{n}$, Boll. Un. Mat. Ital. A 11 (1997), 237-252.

[6] E.M. Stein, Harmonic analysis: Real-variable Methods, Orthogonality, and Oscillatory Integrals, Princeton University Press, Princeton, New Jersey, 1993.

Department of Mathematics, Florida State University, Tallahassee, FL 32306

E-mail address: oberlin@math.fsu.edu 